\title{
MONOCRISTAIS E POLICRISTAIS, UMA AVALIAÇÃO DOS MÉTODOS DE OBTENÇÃO E CARACTERIZAÇÃO
}

\author{
AZEVEDO, A. G. S. \\ ${ }^{1}$ Químico graduado pela Universidade do Estado de Minas Gerais - UEMG. Mestre em Física e Química de \\ Materiais pela Universidade Federal de São João del-Rei - UFSJ. Doutorando em Física e Química de \\ Materiais pela Universidade Federal de São João del-Rei - UFSJ \\ Autor correspondente: Adriano Galvão de Souza Azevedo - Rua Vereador Elí Araújo, 321, apt 203 - Fábricas \\ - São João del-Rei/MG. E-mail: adrianogalvao26@yahoo.com.br
}

Recebido em: 30/10/2013 - Aprovado em: 20/12/2013 - Disponibilizado em: 15/01/2014

RESUMO: A produção de cristais é uma área de grande interesse dentro das ciências dos materiais devido a grande representação tecnologia desses materiais. Existem diversas formas de obtenção desses cristais, cada uma contribuindo para a confecção de materiais com diversas cristalografias, morfologias e energias reacionais superficiais. Aplicando-se o método correto e controlando os parâmetros de síntese se torna possível fabricar cristais mono ou policristalinos. Os policristais são de mais fácil obtenção e não requerem grandes controles. Os monocristais requerem reagentes de elevada pureza, controle da temperatura e métodos particulares que possibilitem a sua fabricação. Esse trabalho buscou elucidar dois mecanismos de obtenção de monocristais e as técnicas mais utilizadas na caracterização desses materiais. Os métodos escolhidos foram os de Bridgman e Czochralsky, por se apresentarem como sendo os mais utilizados para a fabricação de monocristais e por apresentar um menor custo operacional quando comparados a outras técnicas conhecidas na literatura.

PALAVRAS-CHAVE: Monocristais, policristais, Bridgman, Czochralsky.

ABSTRACT: The crystal production is an area of great interest in the material sciences technology due to the large representation of these materials. There are several ways to obtain these crystals, each contributing to the preparation of materials with different crystallinity, morphology and surface reaction energies. Applying the correct method and controlling the synthesis parameters becomes possible to fabricate single crystals or polycrystals. The polycrystals are more readily available and do not require large controls. The single crystals require high purity reagents, temperature control and particular methods that enable the manufacture. This study aimed to elucidate two mechanisms for obtaining single crystals and the techniques used to characterize these materials. The methods chosen were those of Bridgman and Czochralsky, by presenting themselves as being the most widely used for the manufacture of synthetic single crystals and present a lower operating cost when compared to other known techniques in the literature.

KEYWORDS: Single crystals, polycrystals, Bridgman, Czochralsky.

\section{Introdução}

A busca por materiais que contribuam para um desenvolvimento dos setores tecnológicos é de grande importância para pesquisa nas áreas de estudo dos materiais. Pesquisadores trabalham com alterações estruturais, que são acompanhadas de mudanças das características dos materiais desejados (WANG et al., 2009).

A obtenção de novos materiais ou a síntese controlada de materiais já conhecidos e tecnologicamente aplicados são uma das grandes formas de se melhorar as pesquisas nas áreas. $\mathrm{O}$ presente trabalho busca o entendimento das diferenças entre os monocristais e 
os policristais. As diferentes aplicações dos cristais nas diferentes áreas e os diferentes métodos de obtenção dos mesmos (WANG et al., 2009).

As formas de obtenção são conhecimentos de grande importância, pois, as variações de parâmetros de síntese influenciam na microestrutura do produto final. As formas de caracterização dos materiais monocristalinos e policristalinos são ferramentas de grande ajuda para o entendimento e na melhoria das sínteses, pois, ajudam a elucidar as estruturas obtidas após os experimentos e consequentemente a buscarmos uma melhor rota para síntese do material de interesse visando à eficiência energética e reacional.

\section{Monocristais e Policristais}

A ordenação dos átomos ou partículas em um sistema que se encontra no estado líquido é maior do que a ordenação das partículas no estado gasoso e menor do que os átomos no estado sólido. A ordenação das partículas é devido à energia presente no sistema, que se altera ao passarmos de um estado físico da matéria para outro. A energia cinética das partículas é a energia que mede a movimentação dos átomos dentro de um sistema, e a quantificação da energia cinética total presente após alguma reação física e ou química pode ser o suficiente para deduzirmos o ordenamento final das partículas e seu estado físico final (DALL'ANTONIA et al., 1999).

As estruturas dos sólidos se caracterizam por permitir uma mínima movimentação dos átomos constituintes, apenas vibrações são permitidas para tais ordenações. A diminuição da temperatura de um sistema que se encontra em um estado líquido faz com que as os átomos se aproximem uns dos outros, permitindo que interações ocorram entre estes e uma consequente nucleação faça com a formação de cristais seja possível. A nucleação da origem ao crescimento de cristais que se formam a partir de uma diminuição do volume do líquido - os átomos perdem a energia para o universo e se agregam para formarem os cristais - e independentes uns dos outros crescem em direções onde a energia interna seja consideravelmente menor (TREMPA et al., 2012). A formação de cristais que crescem sem um controle das direções cristalográficas dá origem a estruturas desordenadas, onde várias direções de crescimento são encontradas e tamanhos relativos dos grãos formados são dependentes dos controles durante a síntese. 
Ao término da solidificação os cristais que possuem diferentes orientações cristalográficas se ordenam em estruturas tridimensionais e formam os conhecidos sólidos policristalinos. Policristais é a denominação dada para sólidos onde diferentes orientações se encontram presentes, dando ao material sólido diferentes propriedades físicas, químicas e biológicas (ATKINS, 2006).

A formação de sólidos cristalinos com orientações bem definidas e únicas nos cristais (monocristais) é possível a partir do controle de parâmetros como temperatura, concentração dos reagentes, pureza dos reagentes, pressão, tempo de síntese, entre outros (WANG et al., 2009). A preparação de compostos onde os contornos de grãos são inexistentes se demonstra em grande crescimento para o desenvolvimento científico-tecnológico de várias áreas industriais.

A utilização de monocristais artificiais de elevada pureza para aplicações em dispositivos eletrônicos demanda de conhecimento dos mecanismos unitários de obtenção dos compostos sólidos, podendo se controlar os parâmetros essenciais para a formação de cristais de alta perfeição estrutural e grande resposta às necessidades de aplicação, que podem ser relacionadas:
- Circuitos integrados eletrônicos: $\mathrm{Si}, \mathrm{Ge}, \mathrm{GeAs}$, etc.

- Circuitos integrados óticos: $\mathrm{LiNbO}_{3}, \mathrm{LiTaO}_{3}$.

- Memórias óticas: $\mathrm{LiNbO}_{3}, \mathrm{Fe}$, $\mathrm{Bi}_{12} \mathrm{TiO}_{20}$.

- Lasers de estado sólido: GaAs, $\mathrm{Al}_{2} \mathrm{O}_{3}, \mathrm{Y}_{3} \mathrm{Al}_{5} \mathrm{O}_{12}$, entre outros.

- Defletores óticos e guias de ondas: $\mathrm{LiNbO}_{3}$.

Materiais que apresentam estruturas monocristalinas podem ser obtidos por diferentes técnicas que, variam de acordo com a solubilidade do material e de acordo com a sua posterior aplicação. As técnicas de obtenção de monocristais, como já dito, requerem o controle minucioso da síntese para que o cristal possa ter uma cinética de crescimento adequada. Os cristais que são obtidos pelos métodos de crescimento, não possuem grandes tamanhos devido à dificuldade de aplicação dos processos e dos controles adequados.

As diferentes técnicas de obtenção de sólidos monocristalinos estão relacionadas com as condições termodinâmicas impostas durante a síntese. A temperatura, concentração dos reagentes e pressão devem ser cuidadosamente controlados para que os 
átomos presentes na fase líquida possam realizar interações e consequentemente formar retículos cristalinos que se propagam sem alterações por todo o restante do material (NILSO, BARELLI ; MATILDES; BENEDETT, 2003).

Dentre os métodos utilizados para a obtenção dos cristais de interesse, serão citados nesse trabalho dois de maior importância devido a suas facilidades de implantação e custo. Os métodos são conhecidos como: Czochralski e o método de Bridgman. Os métodos se baseiam no crescimento de cristais a partir de soluções que contenham os átomos de interesse (DALL'ANTONIA et al., 1999).

O processo de Czochralsky é um método muito utilizado para obtenção de monocristais de maiores tamanhos, utilizados nas indústrias de eletrônica e ótica. A técnica se baseia na mistura de reagentes de partida - 99,99\% de pureza - dentro de recipientes adequados (cadinhos) e no posterior aquecimento até que todos os reagentes se fundam $\mathrm{A}$ técnica de czochralsky utiliza uma semente para que o cristal se forme na orientação desejada. A semente é presa por um fio e é mergulhada e girada dentro do material fundido de forma que a deposição se dê na superfície da semente e a solidificação do cristal segue a orientação presente na semente.

O controle da taxa de mergulho, temperatura e velocidade de rotação da semente devem ser controladas para que os cristais possam crescer com o menor número de defeitos possíveis, apresentando melhores resultados em utilizações tecnológicas. O controle da atmosfera onde a reação de crescimento ocorre é de grande importância devido à natureza reativa do líquido fundido, sendo necessária a utilização de uma atmosfera inerte $\left(\mathrm{N}_{2}, \mathrm{Ar}\right)$ ou a criação de vácuo. Uma das desvantagens do método de czochralsky é o crescimento de cristais a partir de reagente voláteis, que apresentam pequenas pressões de vapor à temperatura de fusão e acabam por causar alterações estequiométricas dos cristais devido à volatilização e cristalização nas partes superiores do cadinho.

As temperaturas das câmaras são cuidadosamente controladas com o intuito de se obter pequenas diferenças entre as temperaturas de fusão e solidificação dos reagentes. A ampola é movimentada por dentro da câmara em uma velocidade (v) e devido ao gradiente de temperatura existente na câmara, o reagente precursor se funde primeiramente, servindo de posterior 
Tabela 1-Descrição de obtenção, vantagens e desvantagens dos métodos de crescimento de monocristais. Adaptado de (DALL'ANTONIA et al., 1999)

\begin{tabular}{|c|c|c|c|}
\hline Método & Descrição & Vantagens & Desvantagens \\
\hline $\begin{array}{c}\text { Rota de } \\
\text { Bridgman- } \\
\text { stockbarger }\end{array}$ & $\begin{array}{l}\text { O material puro é fundido em gradiente } \\
\text { térmico cuja variação depende da } \\
\text { condutividade térmica do material a ser } \\
\text { cristalizado, em atmosfera inerte } \\
\text { dentro um cadinho cilíndrico } \\
\text { (usualmente de grafite) terminado por } \\
\text { uma ponta cônica. }\end{array}$ & $\begin{array}{l}\text { Crescimento } \\
\text { de cristais com } \\
\text { grandes } \\
\text { massas }\end{array}$ & $\begin{array}{l}\text { Contaminação pelo } \\
\text { material de cadinho }\end{array}$ \\
\hline $\begin{array}{c}\text { Rota de } \\
\text { czocharalski } \\
\text { (também } \\
\text { denominada Rota } \\
\text { de Tração) }\end{array}$ & $\begin{array}{c}\text { Essa rota consiste em descer um } \\
\text { germe monocristalino, animado por um } \\
\text { movimento de rotação, até tocar a } \\
\text { superfície do material fundido. Em } \\
\text { seguida o material é verticalmente } \\
\text { removido, arrastando o restante do } \\
\text { material em forma de monocristal que } \\
\text { se solidifica na orientação do germe } \\
\text { utilizado. }\end{array}$ & $\begin{array}{c}\text { Elevada } \\
\text { velocidade de } \\
\text { crescimento }\end{array}$ & $\begin{array}{l}\text { Contaminação pelo } \\
\text { material do cadinho. } \\
\text { Grande massa mínima } \\
\text { de material fundido }\end{array}$ \\
\hline
\end{tabular}

semente para todo o restante do material que se solidificará. A ampola é mantida sob vácuo e posterior vedação, o que se torna um método viável para a produção de cristais a base de reagentes voláteis. Cada técnica de crescimento de cristais possui sua vantagem e sua desvantagem, que estão relacionadas de modo informativo na tabela 1 .

Vários trabalhos têm sido realizados com intenção de se entender os mecanismos envolvidos nos processos de obtenção de monocristais artificiais. STREICHER et al (2010) realizaram pesquisas com crescimento de monocristais de $\mathrm{GaSb}$ pelo método de Czochralsky para aplicação em lasers infravermelho, fotodiodos $\mathrm{e}$ conversores termofotovotáicos. Os autores utilizaram um cristal de orientação cristalográfica conhecida como semente de crescimento. Após o contato sólido/líquido a semente foi puxada com velocidade controlada e os monocristais do semicondutor foram sendo obtidos. O processo foi realizado a uma temperatura de $720^{\circ} \mathrm{C}$ e em atmosfera de argônio.

Um cristal <111> de GaSb foi utilizado como semente e o controle da velocidade de puxamento e rotação foram controlados manualmente. Utilização de microscópio ótico demonstrou o aparecimento de "pits" nas regiões próximas a semente. Os autores relatam que tais deformações podem ser resultados das tensões causadas durante $\mathrm{o}$ mergulho $\mathrm{da}$ semente no material fundido para a realização da cristalização.

$\mathrm{Na}$ figura 1 temos os resultados das análises realizadas por microscopia ótica dos monocristais obtidos. Nilso et al (2003) trabalharam com a construção de um dispositivo para obtenção de monocristais de ligas ternárias de cobre. 


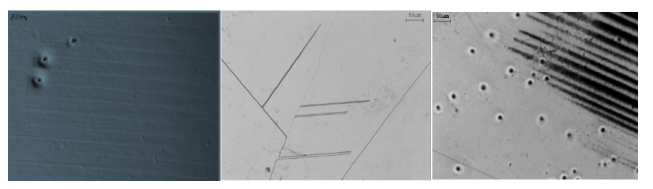

Figura 1 - Microscopia ótica. (a) Estrias (b) Maclas (c) Pits e discordâncias. Adaptado de (STREICHER; DEDAVID, 2010)

A técnica utilizada por esses autores foi a de Bridgman devido às características estruturais que se pretendia preservar nos cristais para a sua posterior utilização. $\mathrm{O}$ trabalho teve foco na obtenção de monocristais de ligas do tipo CuZnAl que diferentemente dos sólidos policristalinos, apresentam um aumento nas propriedades físicas, principalmente nas propriedades mecânicas, que podem chegar a suportar valores durante os ensaios de tração de até $800 \mathrm{Mpa}$ (NILSO, BARELLI; MATILDES; BENEDETT, 2003). Os autores utilizaram ligas policristalinas à base de cobre para construção de eletrodos para estudos ligados a corrosão. O grupo constatou que a utilização de eletrodos construídos a partir de policristais é de difícil entendimento dos processos de natureza corrosiva devido à complexidade das superfícies existente nos eletrodos policristalinos. Essa complexidade é proveniente das diferenças de sítios de adsorção nas superfícies de cristais com diferentes orientações cristalográficas

(DALL'ANTONIA et al., 1999).

A construção de eletrodos utilizando monocristais facilita o trabalho dos pesquisadores devido sua única orientação cristalográfica. Os monocristais apresentam uma única orientação cristalina em todo o sólido e consequentemente respondem a reações químicas de formas diferentes em diferentes orientações cristalográficas, podendo se obter um melhor arranjo atômico para cada aplicação (NILSO, BARELLI ; MATILDES; BENEDETT, 2003). A obtenção dos monocristais pelo método de Bridgman foi dado a partir da obtenção de policristais de CuZnAl de pureza superior a 99,99\%. Os cristais foram posteriormente colocados dentro de um cadinho de quartzo onde se foi criado um vácuo e a vedação foi realizada com auxílio de um maçarico, deixando uma ponta para a fixação do fio de suporte do cadinho. $\mathrm{O}$ cadinho com o material foi colocado dentro do dispositivo criado acima da temperatura de fusão da liga de $\mathrm{CuZnAl}$ e mantido a está temperatura por uma hora para se garantir a fusão de todo material contido dentro do recipiente de quartzo. Após a constatação da mudança de estado do material foi acionado o dispositivo para que o 
cadinho começasse a descer em direção onde a temperatura se encontrava abaixo da temperatura de solidificação. O controle da velocidade e o controle das vibrações ou movimentos do cadinho foram delicadamente supervisionados, uma vez que qualquer choque mecânico pode alterar as orientações de crescimento dos monocristais (NILSO, BARELLI ; MATILDES; BENEDETT, 2003). Os monocristais foram retirados dos cadinhos de quartzo e foram submetidos a técnicas de caracterização, onde pôde ser confirmado a obtenção de apenas monocristais de CuZnAl, como apresentado na figura 2 .

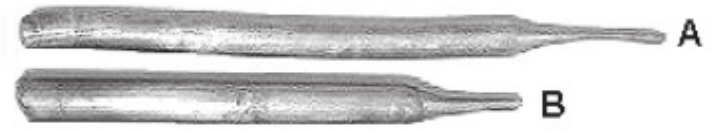

Figura 2 - Fotografia dos monocristais obtidos pela técnica de Bridgman. (a) monocristais de $\mathrm{CuZnAl}$ fase beta (b) monocristais de $\mathrm{CuZnAl}$ de fase martensítica. Adaptado de (NILSO, BARELLI ; MATILDES; BENEDETT, 2003)

Por outro lado, a produção de policristais não requer um controle tão minucioso dos processos de crescimento. $\mathrm{O}$ policristal pode ser obtido pelo descumprimento dos controles de processos do crescimento de monocristais. Ao contrário de se obter uma estrutura que possui apenas um arranjo cristalino, o crescimento de policristais tem o seu foco na produção de sólidos com orientações cristalinas distintas dentro do mesmo material.

Nilso et al (2003) relatam que medidas realizadas com eletrodos produzidos a partir de policristais não apresentam um grande índice de confiabilidade e que variam desde laboratórios a análises de rotina. Tais discrepâncias nos resultados obtidos são devido aos diferentes arranjos cristalográficos presentes no material e que apresentam características físicas e químicas muito diferentes dos monocristais (Willian D. Callister, 2011).

De acordo com Callister (2011), os metais que apresentam maiores números de cristais crescidos e grãos formados, possuem maiores valores de resistência mecânica durante os ensaios. A resistência desses materiais é devido aos mecanismos de escorregamento que ocorrem de diferentes formas, sendo mais fáceis em monocristais e são dificultadas em policristais.

Os escorregamentos nos metais policristalinos sofrem resistência ao passarem de um grão formado para outro adjacente, devido às diferentes 
orientações

das

estruturas

cristalográficas. Mesmo que um grão esteja orientado em uma direção que favorecerá o escorregamento, ao se chegar aos contornos de grão fronteiras entre dois grãos com diferentes orientações cristalográficas os escorregamentos sofrem interferências devido à descontinuidade existente entre nos contornos. Assim, maiores valores de tensão são necessários para dar continuidade à falha nesses materiais (ASM, 2012).

Os átomos fronteiriços dos grãos adjacentes não estão ligados da mesma forma que os átomos presentes em regiões mais centralizadas do cristal, o que promove diferenças nas energias nessas regiões e podem ser responsáveis por defeitos conhecidos como defeitos de superfície (Willian D. Callister, 2011).

RIBEIRO et al (2007) avaliaram defeitos em amostras produzidas com diferentes policristais de ligas metálicas e concluíram que diferentes fazes existentes no material - fase austenítica e martensíntica - modificam os valores de resistência e alteram as possibilidades de aplicação dos materiais em diversas áreas.

A aplicação de policristais não se volta somente para a produção de materiais metálicos. A produção de uma variedade de materiais cerâmicos que apresentam tais características é foco de estudos dentre muitos pesquisadores. BRAULIO et al (2010) avaliaram a influencia de vários parâmetros durante a produção de cerâmicas refratárias a base de $\mathrm{MgO}$ e $\mathrm{Al}_{2} \mathrm{O}_{3}$. Os autores modificaram os tamanhos de partículas da matéria prima precursora $(\mathrm{MgO})$ e concluíram que tais mudanças alteram o tamanho dos grãos obtidos no final do processo de produção. A mudança na temperatura de processamento de materiais cerâmicos é outro fator de grande importância durante a obtenção dos materiais. BRAULIO et al (2010) modificaram a temperatura de obtenção dos materiais cerâmicos e observaram que a cinética da reação é influenciada pela temperatura. A análise por DRX (difração por Raios X) confirmou o aparecimento de picos referentes a espécies que inexistentes nos diagrama de fases do material. Eles relacionaram tal ocorrência com a cinética da reação, que apresenta um decréscimo diretamente proporcional à diminuição da temperatura de sinterização. Em temperaturas mais brandas e com reagentes de partida com de maior granulometria é observado também a presença de outras fases - Alumina tubular - que são subprodutos de reações que não se completaram na sua 
totalidade. Em $1500^{\circ} \mathrm{C}$ os resultados de DRX detectam picos referentes às espécies fosterita e monticelita, que estão relacionadas a pouca reatividade dos reagentes com maiores tamanhos de grãos (BRAULIO et al., 2010).

Processos onde as temperaturas de sinterização são mais altas apresentam materiais mais densos devido à difusão dos átomos. Os mecanismos de sinterização fazem com que os tamanhos dos grãos aumentem e consequentemente tornem o material mais frágil (Willian D. Callister, 2011).

CINTRA et al (2010) avaliaram a granulometria da matéria prima adequada para a preparação de espinélios com aplicação em fornos refratários utilizados nas indústrias siderúrgicas. Eles avaliaram da mesma forma que BRAULIO et al (2010), as alterações cristalográficas causadas pela utilização de diferentes tamanhos das partículas de $\mathrm{MgO}$ na síntese dos corpos cerâmicos e a influência dessas alterações estruturais nos resultados dos ensaios mecânicos realizados. Eles constataram que a utilização de partículas menores aumenta a taxa de reação e formação de espinélios. Os autores avaliaram juntamente a influência da temperatura de sinterização dos refratários e concluíram que em temperaturas mais elevadas têm-se uma perda da resistência mecânica do material. Tal perda pode estar relacionada com a formação de outras espécies diferentes das esperadas devido reações químicas incompletas, que apresentam volumes maiores e acabam por gerar tensões $\mathrm{e}$ consequentemente, a formação e propagação de microtrincas na estrutura do material cerâmico, o que diminui consideravelmente a sua resistência mecânica (CINTRA et al., 2010).

Outro fator que está relacionado com o aumento da temperatura de calcinação, o consequente processo de sinterização e a diminuição da resistência mecânica do material é o aumento do tamanho dos grãos. Grãos maiores crescem "engolindo" grãos de menores tamanhos. O crescimento dos grãos dentro de um material favorece a propagação de trincas em um plano de maior densidade planar, uma vez que em todo o grão a sua orientação cristalográfica é similar, as trincas podem se propagar sem impedimentos por toda a área do grão, diminuindo os valores de resistência nos ensaios mecânicos (Willian D. Callister, 2011). 


\section{Métodos de Caracterização de Monocristais e Policristais}

Os materiais obtidos pelos diferentes métodos de crescimento são, posteriormente, caracterizados por métodos que são capazes de observar as ordenações estruturais (DRX), vibrações correspondentes de grupamentos químicos e consequentemente a presença de impurezas podem ser detectadas pela espectroscopia no Infravermelho com transformada de Fourie (IVTF) e para avaliar a morfologia do material utilizase a microscopia eletrônica de varredura (MEV) e a microscopia ótica (MO).

Esses são alguns dos métodos capazes de caracterizar materiais sólidos cristalinos.

Vários autores utilizaram a difração de Raios X para realizarem a caracterização de seus materiais, sejam eles monocristalinos ou policristalinos (COSTA et al., 2009; PEREZ; VILLULLAS; GONZALEZ， 1997; RIBEIRO; PRADO, 2010; WANG et al., 2009). Tal técnica consegue, por meio da reflexão dos Raios X, avaliar as periodicidades das redes cristalinas $\mathrm{e}$ consequentemente a existência de fases diferentes no cristal, como demonstrado na figura 4. Essa técnica é de grande importância no estudo da produção e caracterização de mono e policristais.

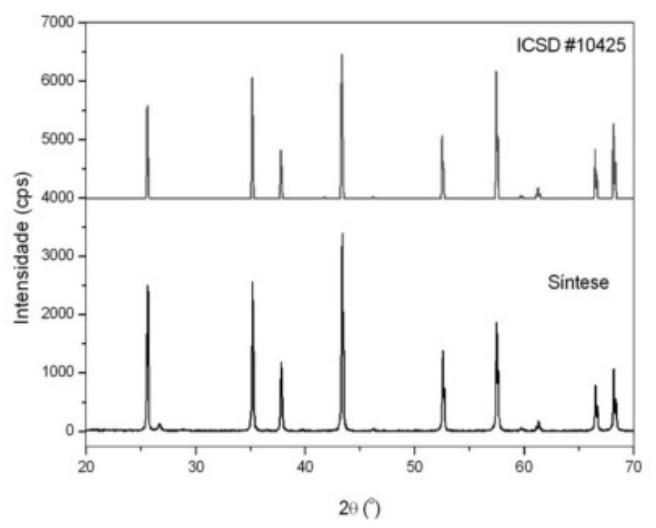

Figura 4 - Difratograma de Raios X obtido de uma das amostras de rubi sintetizada por (RIBEIRO; PRADO, 2010) e sua comparação com dados da literatura

A microscopia eletrônica de varredura é uma técnica capaz de produzir imagens de alta resolução de superfícies. Devido às altas resoluções criadas pelo equipamento, várias deformidades existentes na superfície podem ser avaliadas e são de grande utilidade na avaliação de crescimento de cristais. Através da aceleração de elétrons que se chocam com a superfície da amostra o equipamento gera as imagens através dos sinais que são "Arrancados" dos átomos presentes no material (elétrons secundários, Raios X característicos, radiação IV, entre outros.). Os dados obtidos são transformados em imagens a partir da transcodificação da energia dos elétrons ejetados da superfície da amostra. 
CINTRA et al (2010) e RIGO et al (2007) utilizaram da tecnologia proveniente do microscópio eletrônico de varredura como uma das caracterizações dos materiais sintetizados. O que de acordo com os autores é de grande importância para a área de obtenção materiais a utilização dessa técnica onde, a estrutura superficial é de grande interesse.

(RIGO et al (2007) COSTA et al (2009) durante a síntese de cristais de hidroxiapatita $\left(\mathrm{Ca}_{10}\left(\mathrm{PO}_{4}\right)_{6}(\mathrm{OH})_{2}\right)$ para a utilização em implantes ósseos, aplicaram a espectroscopia no infravermelho na caracterização do material de interesse.
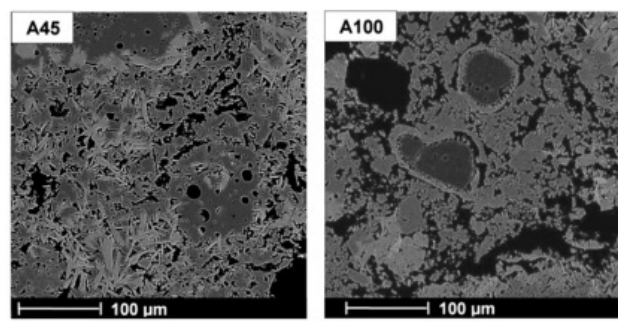

Figura 5 -: Imagens obtidas por microscopia eletrônica de varredura de concretos contendo tamanhos de grão de $\mathrm{MgO}$ distintos, após queima a 1500 o $\mathrm{C}$ por $5 \mathrm{~h}$. Adaptado de (CINTRA et al., 2010).

Os autores observaram nos espectros a presença de outros grupamentos, diferentes dos encontrados nos cristais de HAp (hidroxiapatita).

A presença de grupamentos $\mathrm{CO}_{3}{ }^{2-}$ (íon carbonato) foi observada e correlacionada com a entrada do grupamento através de impurezas dos reagentes ou contaminações atmosféricas. Como a utilização de materiais in vivo requer o máximo de pureza possível, a técnica de espectroscopia do infravermelho se torna essencial para a caracterização de materiais com essas posteriores aplicações.

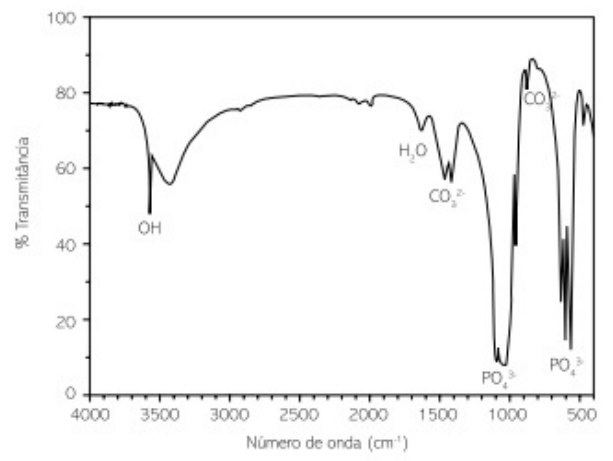

Figura 6 - Espectroscopia no infravermelho do pó de hidroxiapatita mostrando a presença de impurezas $\left(\mathrm{CO}_{3}{ }^{2-}\right)$. Adaptado de (RIGO; GEHRKE; CARBONARI, 2007).

\section{Conclusão}

Os cristais sejam eles naturais ou artificiais, apresentam características cristalográficas que os diferenciam uns dos outros e que modificam suas 
propriedades físicas e químicas. Pesquisas relacionadas às rotas $\mathrm{e}$ métodos de obtenção de cristais têm se intensificado nos últimos anos devido as mais diversas áreas de aplicação tecnológicas desses materiais. $\mathrm{O}$ controle durante a preparação dos cristais é de extrema importância uma vez que as modificações de pequenos parâmetros alteram a estrutura do produto final, o que pode ser observado através de técnicas de caracterização de materiais disponíveis no mercado.

\section{Referências Bibliográficas}

ATKINS, P. Físico-Química. Oitava Edi ed. [s.l: s.n.]. p. 330-338

BRAULIO, M. A. L. et al. Variação do tamanho de grão da magnésia : um exemplo da complexidade de cerâmicas refratárias ( Changing the magnesia grain size : an example of. v. 56, p. 222-227, 2010.

CINTRA, G. B. et al. Tamanho de grão do $\mathrm{MgO}$ e seus efeitos na resistência ao choque térmico de concretos refratários espinelizados in-situ ( $\mathrm{MgO}$ grain size and the thermal shock performance of in-situ spinel. v. 56, p. 15-22, 2010.

COSTA, A. C. F. M. et al. Hidroxiapatita : Obtenção, caracterização e aplicações. Revista Eletrônica de Materiais e Processos, v. 3, p. 29-38, 2009.

DALL'ANTONIA, L. H. et al. Metodologia para o crescimento de esferas monocristalinas de metais nobresQuímica Nova, set. 1999.

NILSO, BARELLI ; MATILDES, B.; BENEDETT, A. V.

DESENVOLVIMENTO DE UM

DISPOSITIVO PARA OBTENÇÃO

DE MONOCRISTAIS DE LIGASS À

BASE DE COBRE. v. 26, n. 5, p. 757762, 2003.

PEREZ, J.; VILLULLAS, H. M.; GONZALEZ, E. R. NOTA TÉCNICA PREPARAÇÃ̃ E

CARACTERIZAÇÃO DE ELETRODOS MONOCRISTALINOS. QUÍMICA NOVA, v. 20, n. 5, p. 4548, 1997.

RIBEIRO, G. DA S.; PRADO, R. J. Quim. Nova. Química Nova, v. 33, n. 5, p. 1104-1108, 2010.

RIBEIRO, M.; GONZALEZ, B. M. Metalurgia Física Efeito do gradiente de martensita induzida por deformação sobre o fenômeno de delayed cracking em aços inoxidáveis austeníticos. v. 60, n. 1, p. 169-173, 2007.

RIGO, E. C. DA S.; GEHRKE, S. A.; CARBONARI, M. Síntese e caracterização de hidroxiapatita obtida pelo método da precipitação.

bionnovation.com.br, p. 39-50, 2007.

STREICHER, M.; DEDAVID, B. A. Crescimento e Caracterização de Cristais de GaSb por Czochralski com Líquido Encapsulante. v. 3, p. 578-580, 2010.

TREMPA, M. et al. Mono-crystalline growth in directional solidification of silicon with different orientation and splitting of seed crystals. Journal of Crystal Growth, v. 351, n. 1, p. 131140, 2012. 
WANG, Y. et al. Hydrothermal

synthesis of single-crystal BaTiO3

dendrites. Materials Letters, v. 63, n.

2, p. 239-241, jan. 2009. 